\title{
High-speed imaging upgrade for a standard sample scanning atomic force microscope using small cantilevers
}

\author{
Jonathan D. Adams, Adrian Nievergelt, Blake W. Erickson, Chen Yang, Maja Dukic, and Georg E. Fantner
}

Citation: Review of Scientific Instruments 85, 093702 (2014); doi: 10.1063/1.4895460

View online: https://doi.org/10.1063/1.4895460

View Table of Contents: http://aip.scitation.org/toc/rsi/85/9

Published by the American Institute of Physics

\section{Articles you may be interested in}

A miniaturized, high frequency mechanical scanner for high speed atomic force microscope using suspension on dynamically determined points

Review of Scientific Instruments 86, 113703 (2015); 10.1063/1.4935584

A high frequency sensor for optical beam deflection atomic force microscopy

Review of Scientific Instruments 82, 043705 (2011); 10.1063/1.3575322

Development of low noise cantilever deflection sensor for multienvironment frequency-modulation atomic force microscopy

Review of Scientific Instruments 76, 053704 (2005); 10.1063/1.1896938

A versatile atomic force microscope integrated with a scanning electron microscope

Review of Scientific Instruments 88, 053704 (2017); 10.1063/1.4983317

Digitally controlled analog proportional-integral-derivative (PID) controller for high-speed scanning probe microscopy

Review of Scientific Instruments 88, 123712 (2017); 10.1063/1.5010181

Wideband low-noise optical beam deflection sensor with photothermal excitation for liquid-environment atomic force microscopy

Review of Scientific Instruments 80, 023707 (2009); 10.1063/1.3086418

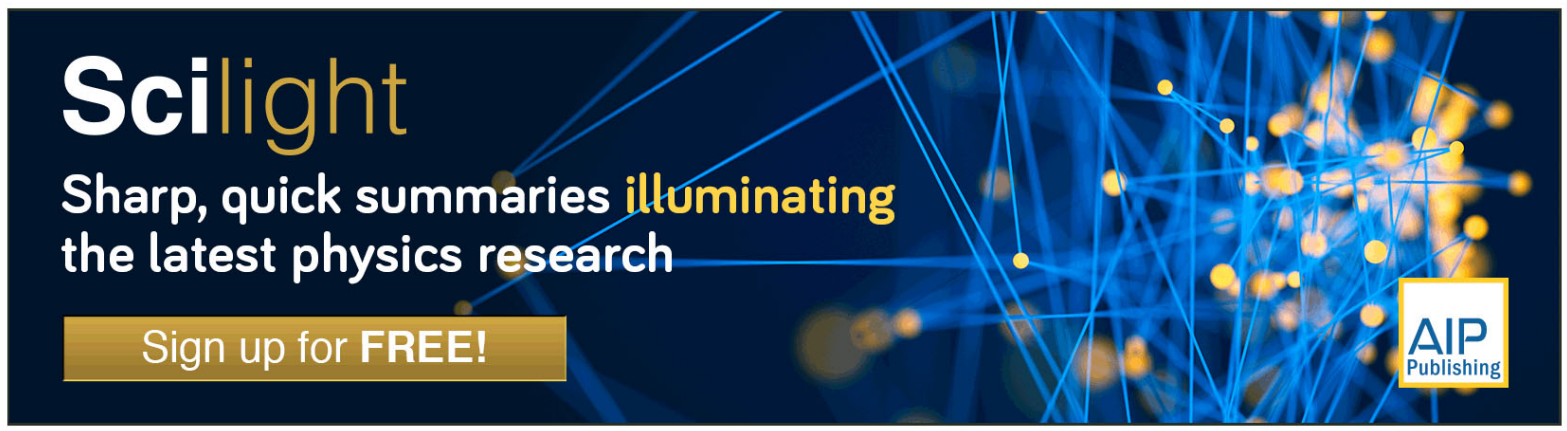




\title{
High-speed imaging upgrade for a standard sample scanning atomic force microscope using small cantilevers
}

\author{
Jonathan D. Adams, Adrian Nievergelt, Blake W. Erickson, Chen Yang, Maja Dukic, \\ and Georg E. Fantner ${ }^{\text {a) }}$ \\ Ecole Polytechnique Fédérale de Lausanne, Lausanne, Switzerland
}

(Received 12 April 2014; accepted 29 August 2014; published online 19 September 2014)

\begin{abstract}
We present an atomic force microscope (AFM) head for optical beam deflection on small cantilevers. Our AFM head is designed to be small in size, easily integrated into a commercial AFM system, and has a modular architecture facilitating exchange of the optical and electronic assemblies. We present two different designs for both the optical beam deflection and the electronic readout systems, and evaluate their performance. Using small cantilevers with our AFM head on an otherwise unmodified commercial AFM system, we are able to take tapping mode images approximately 5-10 times faster compared to the same AFM system using large cantilevers. By using additional scanner turnaround resonance compensation and a controller designed for high-speed AFM imaging, we show tapping mode imaging of lipid bilayers at line scan rates of $100-500 \mathrm{~Hz}$ for scan areas of several micrometers in size. (C) 2014 AIP Publishing LLC. [http://dx.doi.org/10.1063/1.4895460]
\end{abstract}

\section{INTRODUCTION}

Since its introduction, atomic force microscopy (AFM) has developed into a ubiquitous tool for imaging and manipulating objects at the nanometer scale. In AFM, the mechanical characteristics of the cantilever probe strongly determines the overall performance of the microscope. From the first optically-detected cantilevers, handmade from wire with dimensions of order $1 \mathrm{~mm},{ }^{1}$ to the latest small cantilevers approaching size limits of optical detection techniques, ${ }^{2-5}$ performance gains have been strongly coupled with size reductions of the cantilever. Reducing the cantilever dimensions reduces the mass and increases the resonance frequency while maintaining the spring constant at reasonable values. Small cantilevers enabled imaging at high speed, ${ }^{4,6-8}$ even on difficult biological samples, ${ }^{9-11}$ and increased force spectroscopy resolution $^{12}$ and pulling rates. ${ }^{3,13}$ In spite of their obvious benefits, AFM systems making use of small cantilevers have only recently become commercially available.

Beyond the cantilever probe, the imaging speed of AFM is limited by the mechanical and electrical bandwidths of the scanner and feedback components. Coupled with small cantilevers, developments in scanner design and improved control algorithms greatly increased AFM imaging speed. These high-speed scanners include counterbalanced scanners, ${ }^{6,14}$ microresonator scan stages, ${ }^{15}$ flexure-based scanners,,${ }^{8,16,17}$ shear piezo scanners, ${ }^{18}$ and MEMS-based scanners. ${ }^{19}$ Highspeed AFM control strategies include cross-coupling cancellation in piezotube scanners, ${ }^{20,21}$ optimized fast amplitude detection and feedback electronics for high bandwidth, ${ }^{6,18}$ dynamic PID, ${ }^{22}$ and model-based $H_{\infty}$ control methods. ${ }^{23,24}$ Although the combination of high bandwidth cantilevers, scanners, and feedback is necessary in combination to image as quickly as possible, using small cantilevers nevertheless provides significant benefits to many otherwise unmodified or minimally modified AFM systems. Figure 1 illustrates the

a) georg.fantner@epfl.ch major mechanical and electrical components, and their measured bandwidths, for a common AFM system (MultiMode AFM with Nanoscope V controller, Bruker Nano Surfaces, Santa Barbara, CA). For tapping-mode imaging in air (the most common AFM imaging mode) the slowest component in the system is the cantilever, which has an imaging bandwidth $B \simeq \pi f_{0} / Q$, where $f_{0}$ is the resonance frequency of the cantilever and $Q$ the quality factor. ${ }^{25}$ For a large tapping-modein-air cantilever with $f_{0} \simeq 300 \mathrm{kHz}$ and $Q \simeq 500$ (OTESPA, Bruker AFM Probes, Camarillo, CA) we measure $B$ to be of order $1 \mathrm{kHz}$. In contrast, the scanner (Model J, Bruker Nano Surfaces) has a measured $z$ resonance at approximately $10 \mathrm{kHz}$, the controller has a maximum PID bandwidth of approximately $80 \mathrm{kHz}$, and the detection electronics (MultiMode Low Noise Head, Bruker Nano Surfaces) have a usable bandwidth of approximately $2 \mathrm{MHz}$.

In this report, we present a design for a compact, modular, and user-friendly small cantilever optical head for scanning-sample AFMs. Our head is designed to easily integrate into a MultiMode AFM system with minimal external instrumentation requirements and minimal change in user experience. ${ }^{26}$ We describe the optical and mechanical architecture of the head and show the performance of our optical beam deflection architecture. We demonstrate the ability to image a challenging sample at speeds much greater than those of the unmodified AFM by replacing only the AFM head and cantilever used for imaging. By adding additional compensation strategy for the scanner dynamics and a high-speed AFM controller, we demonstrate imaging of a supported lipid bilayer in fluid at line scan rates up to $500 \mathrm{~Hz}$ for scan sizes of $2.7 \mu \mathrm{m}$.

\section{SMALL CANTILEVER HEAD DESIGN}

\section{A. Optical design}

The $\simeq 10 \mu \mathrm{m}$ or smaller width of small cantilevers requires a small focal spot size. The width of a beam waist, $w_{0}$, 


\section{(a)}

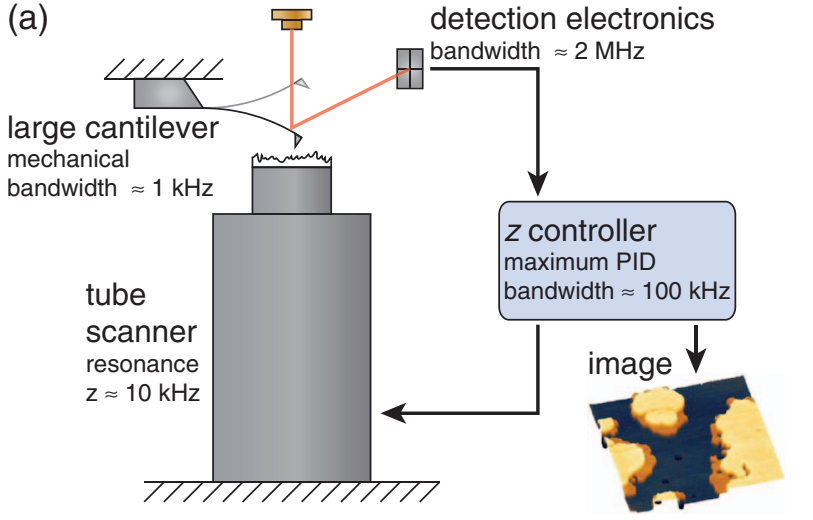

(b)

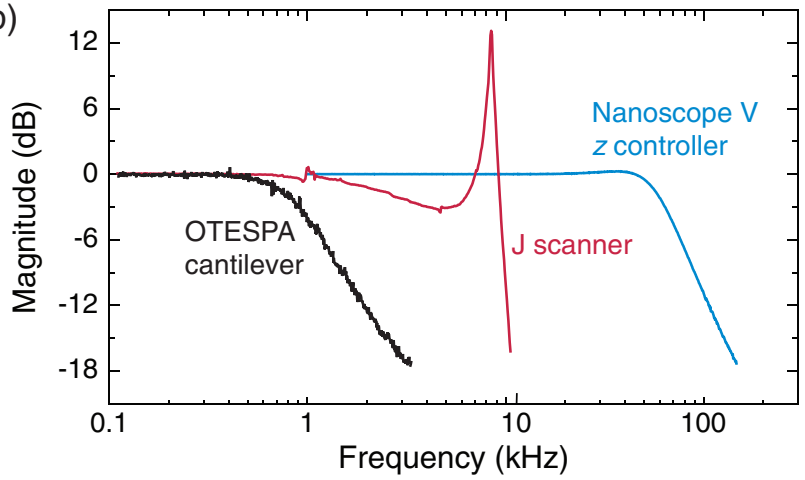

FIG. 1. (a) Major mechanical and electrical components of an AFM system and their typical bandwidths. In the most common AFM imaging mode, tapping mode in air, the detection bandwidth of the cantilever is the slowest component of the system. (b) Bandwidth measurement of the components for a MultiMode AFM system with Nanoscope V controller. The tapping mode mechanical bandwidth of a large AFM cantilever is $\simeq 1 \mathrm{kHz}$, the resonance of the tube scanner occurs at $\simeq 10 \mathrm{kHz}$ and the controller has a maximum PID bandwidth of $\simeq 80 \mathrm{kHz}$.

is related to the numerical aperture of the focusing lens, NA, as $w_{0} \simeq \lambda /(\pi \mathrm{NA})$, where $\lambda$ is the wavelength of the light; therefore, the focusing lens must either be very large in diameter or be placed very close to the cantilever. This proximity typically requires the incident and reflected light paths to pass through the same focusing lens. Two different approaches are used to separate the incident and reflected light paths: polarization-based separation or spatial beam separation. Each approach has relative advantages in terms of cost, complexity, and performance. We have integrated the major optical components into a single monolithic block in our design, permitting interchangeable use of either approach.

Figure 2 illustrates the two different architectures of our optical beam deflection (OBD) assembly. Panel (a) shows the spatial beam separation assembly and panel (b) shows the polarization-based separation assembly. The left parts of panel (a) and (b) show the optical components in isolation along with the approximate extent of the laser light path. The right parts show a section view of the mechanical assembly, with physical placement of the optical components in each module. In either architecture, the laser light is emitted from a diode (HL6355MG, Conrad, Dietlikon, Switzerland) and collimated with an aspheric lens (A390-A, Thorlabs, Newton, $\mathrm{NJ}$ ) and an aperture set slightly larger than the width of the collimated beam to reduce stray reflections. The diode, collimation lens, and aperture are mounted in a collimation (a)

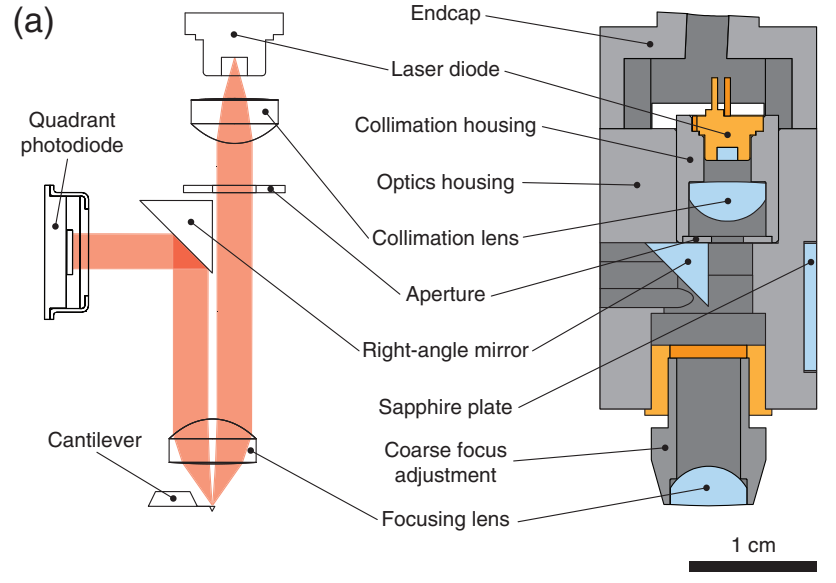

(b)

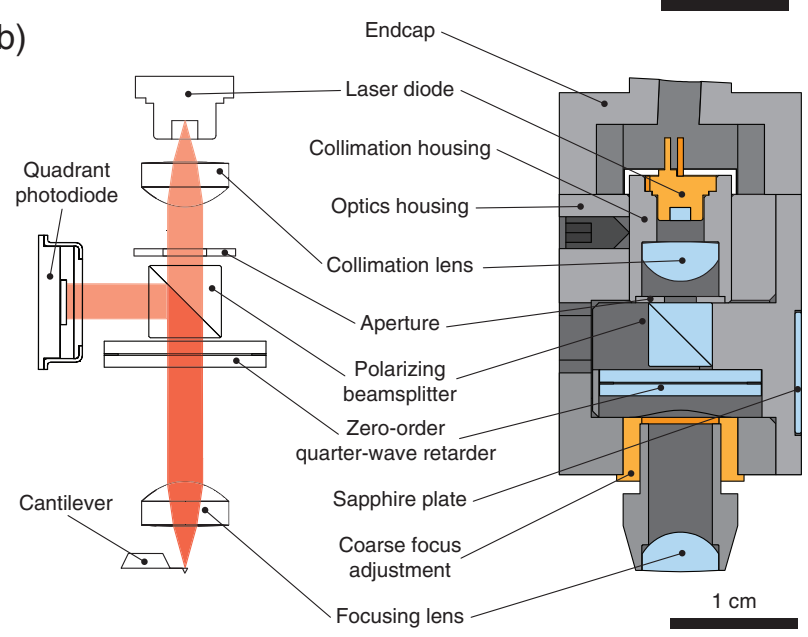

(c)

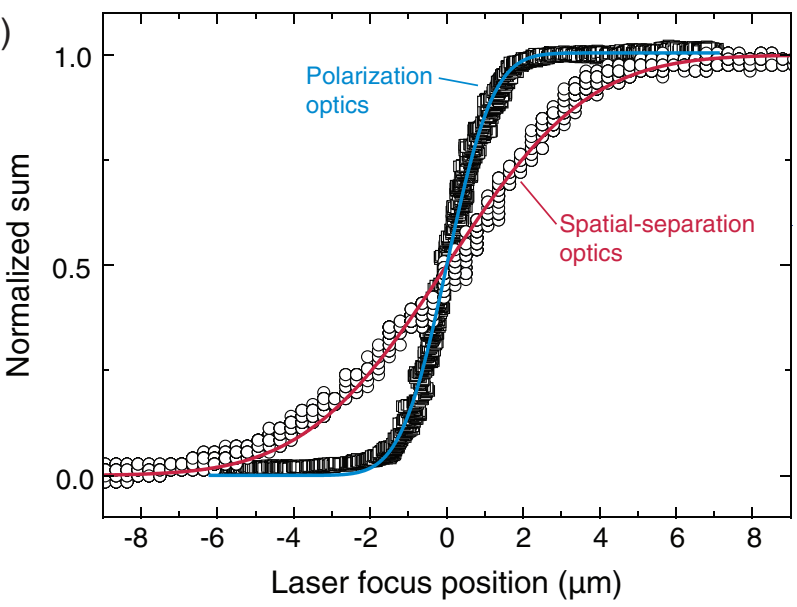

FIG. 2. Schematic of the spatial separation (a) and polarization-based (b) optical beam deflection approaches. The major optical components along with the approximate light path are shown in isolation on the left, and the mechanical layouts of the components in the two OBD assemblies are shown on the right. (c) Measurement of the focal spot size using a knife-edge technique. The $1 / e^{2}$ waist of the spatial separation and polarization-based separation approaches are 5.8 and $1.9 \mu \mathrm{m}$, respectively.

housing for alignment, which is fixed in the optics housing with a set screw. In the spatial beam separation approach (panel (a)), the incident light path is offset axially from the central axis of the focusing lens (A390-A, Thorlabs) by $1.5 \mathrm{~mm}$, such that the focused light is incident on the cantilever at an overall angle. The reflected light returns through same lens on the opposite side of the lens axis and 
is re-collimated. The reflected beam is directed towards a quadrant photodiode (S4349, Hamamatsu, Hamamatsu City, Japan) with a right-angle mirror (MRA05-P01, Thorlabs). In the polarization-based separation approach (panel (b)), the collimated light passes through a polarizing beamsplitter (PBS052, Thorlabs), a zero-order quarter-wave retarder (WPQ05M-633, Thorlabs) and subsequently the focusing lens. The reflected light from the cantilever passes back through the focusing lens and quarter-wave retarder. Proper alignment of the initial beam polarization and quarter-wave retarder allows for effective separation of the reflecting beam from the incident beam at the polarizing beamsplitter. ${ }^{27}$

We measured the $1 / e^{2}$ waist of the focused optical beam of our two optical modules using a knife-edge technique (Figure 2(b)). A laser interferometer (OFV512, Polytec, Waldbronn, Germany) and vibrometer controller (OFV3001, Polytec) tracked the position of the optics block, and by the rule of similar triangles the laser spot, as it was swept transversely across a cantilever (RTESPA, Bruker AFM probes) mounted in the head. We positioned the laser spot longitudinally along the cantilever midway towards the base, well away from the end of the cantilever. The position output of the vibrometer controller and sum signal from the quadrant photodiode were recorded as $x$ and $y$ signals, respectively. The data were fit with a scaled, offset error function to extract the width of the focused spot. The beam waists were measured at $5.8 \mu \mathrm{m}$ and $1.9 \mu \mathrm{m}$ for the spatial beam separation and polarization-based separation approaches, respectively (Figure 2(c)). We attribute the improved focusing of the polarization-based approach to the on-axis orientation of the focusing lens.

\section{B. Mechanical design}

The major mechanical components of our head assembly are shown in Figure 3(a) including the optical beam deflection assembly, the head housing, and a translation stage which mount the head onto the scanner and provide manual translation of the cantilever over the sample. Each component is discussed in detail below.

The OBD assembly, shown in Figure 3(a), is translated as a monolithic unit for positioning the focal spot onto the cantilever. ${ }^{28}$ The OBD assembly is translated with compact differential micrometer screws (DAS110, Thorlabs), which press against sapphire plates (NT43-366, Edmund Optics) inset into the sides of the optics housing (see Figures 2(a) and 2(b)). In our optical design, the aligned position of the OBD assembly is normal to the cantilever, which is tilted at $11^{\circ}$ to the sample normal. The OBD assembly is mounted in the head housing on a flexure spring made out of steel. The resting position of the OBD assembly is set off-axis to the cantilever normal by an angled mounting surface on the optics housing end-cap (Figure 2). When the focal spot is aligned onto the cantilever, the OBD assembly is substantially aligned to the cantilever normal, and the flexure spring exerts a restoring force in both the longitudinal and transverse directions holding the $\mathrm{OBD}$ assembly firmly against the two differential micrometer screws. Fine focusing of the OBD

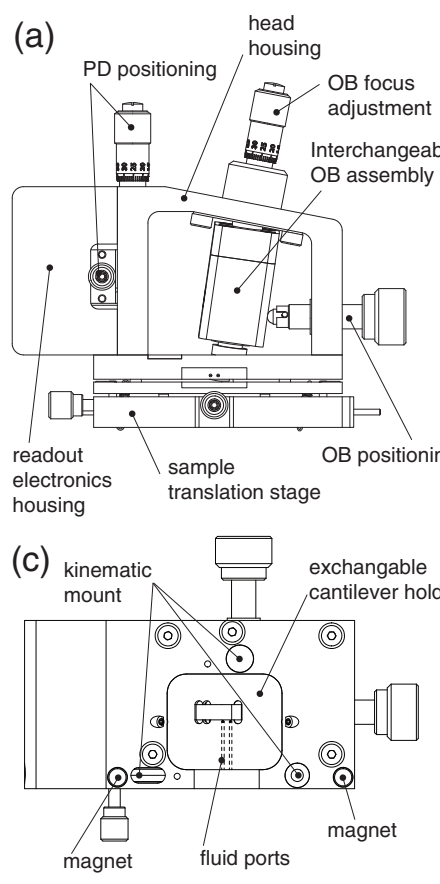

(b) OB focus adjustment

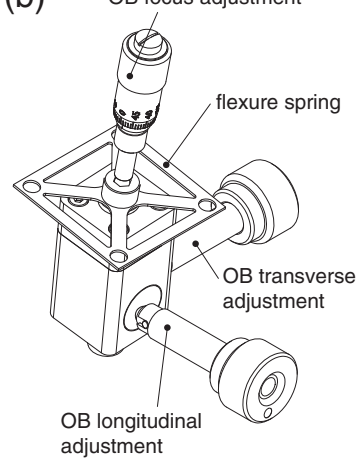

(d)

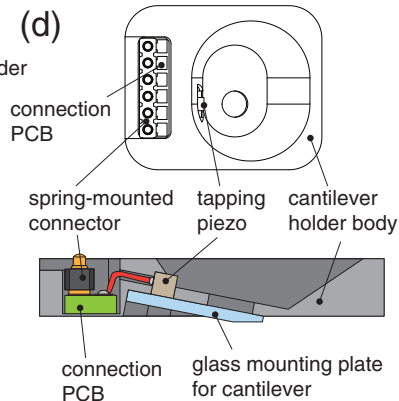

(e)

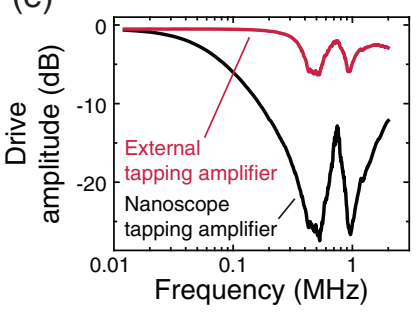

(f)

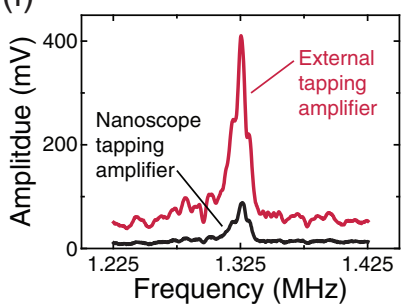

FIG. 3. Technical drawings of the head showing the major features and subassemblies. (a) Front view. (b) Optical beam deflection assembly with focusing and translation adjusters. (c) Bottom view of the head without the translation stage. (d) Section view of the cantilever holder. (e) Performance of the tapping piezo drive (standard drive from the Nanoscope controller and external buffer drive). (f) Excitation of a FastScan A cantilever with the standard Nanoscope and external buffer drive, using a $1 \mathrm{~V}$ drive signal amplitude.

assembly is performed with a micrometer screw (148-205ST, Thorlabs), which presses against a $3 \mathrm{~mm}$ steel ball placed in the hex socket of a M4 socket head cap screw used to fix the OBD assembly onto the flexure spring. A flexible PCB makes the electrical connection between the OBD assembly and the readout electronics in the head housing.

The head housing contains the OBD assembly, the quadrant photodiode (PD), a removable cantilever holder, and the readout electronics. The PD is mounted on a serial two-axis positioning stage allowing for horizontal and vertical positioning. The PD is connected to the readout electronics with a flexible PCB. The head housing is mounted onto the sample translation stage with a kinematic mount. A set of magnets in the head housing and the sample translation stage separated by a small air gap provide retention force to hold the head onto the sample translation stage. The cantilever is held in an exchangeable cantilever holder, which inserts into the base of the head housing and is retained with set screws. Two channels in the cantilever holder provide fluidic access from the front side of the head to the cantilever. 
Figure 3(d) shows a section view of the cantilever holder. The cantilever holder body is made out of aluminum. A glass plate, cut from a piece of glass with anti-reflection coating (48-927, Edmund Optics), sits in a pocket cut at $11^{\circ}$ to the sample normal, and provides a surface for mounting the cantilever. The cantilever is mounted onto the glass plate with Petro Wax (DJB Instruments, Mildenhall, UK). Wax mounting provides both good mechanical coupling and simplified the mechanical design of the canitlever holder. Tapping excitation is provided by a $2 \times 2 \times 2 \mathrm{~mm}$ stack piezo (PL022.30, Physik Instrumente, Karlsruhe, Germany) bonded into a pocket between the glass plate and the cantilever holder body. The assembly is sealed against fluids with epoxy. Electrical connections to the tapping piezo drive are made through the cantilever holder body onto a connection PCB, which then connects to the head housing with spring mounted connectors (811-SS-006-30-08101, Preci-Dip, Delémont, Switzerland). The higher capacitance of the stack piezo compared with the piezo in the MultiMode head is a difficult load for the tapping drive amplifier in the Nanoscope V controller. We therefore have implemented an external high-current buffer (LT1210, Linear Technology, Milpitas, CA) to drive the tapping excitation piezo if the standard drive is insufficient. Figure 3(e) shows the improvement in drive amplitude of the tapping excitation piezo. The external amplifier provides a flatter response and extends the $-3 \mathrm{~dB}$ point of the drive amplitude from $55 \mathrm{kHz}$ to $370 \mathrm{kHz}$. Exciting a small cantilever (FastScan A, Bruker AFM probes) with the external buffer yields a 4.6-fold increase in the peak oscillation amplitude over the standard nanoscope drive (Figure 3(f)).

\section{SIGNAL READOUT}

The two major functions of the signal readout electronics are providing reverse biasing of the quadrant photodiode and current to voltage conversion of the quadrant photodiode signals. We have implemented two different signal readout architectures accomplishing these functions. In the first case, with maximum compatibility with the existing AFM system, and in the second, with high readout bandwidth. In both cases, we reverse bias the quadrant photodiode with a precision voltage reference.

In the high-compatibility readout architecture, we use a transimpedance amplifier to perform the current to voltage conversion of the quadrant photodiode signals. The bandwidth of our readout system is set at $2 \mathrm{MHz}$ in order to maintain highest compatibility with the existing MultiMode system. Our high-bandwidth readout architecture uses a novel translinear photodiode readout circuit which is capable of high bandwidth and low noise performance. ${ }^{29}$ The high bandwidth of this readout approach was tested using a small cantilever (BL-AC10DS-A2, Olympus, Japan) with dimensions $2 \times 9 \mu \mathrm{m}$ and fundamental resonance frequency $\simeq 1.5 \mathrm{MHz}$. The power spectrum of the cantilever thermal deflections was captured on an oscilloscope and calibrated by comparison of the first thermal peak captured on the oscilloscope with the same calibrated thermal peak measured with the MultiMode AFM system. Figure 4(a) shows the thermal peak of the first and second resonances of this cantilever, at 1.4 and $8.8 \mathrm{MHz}$,
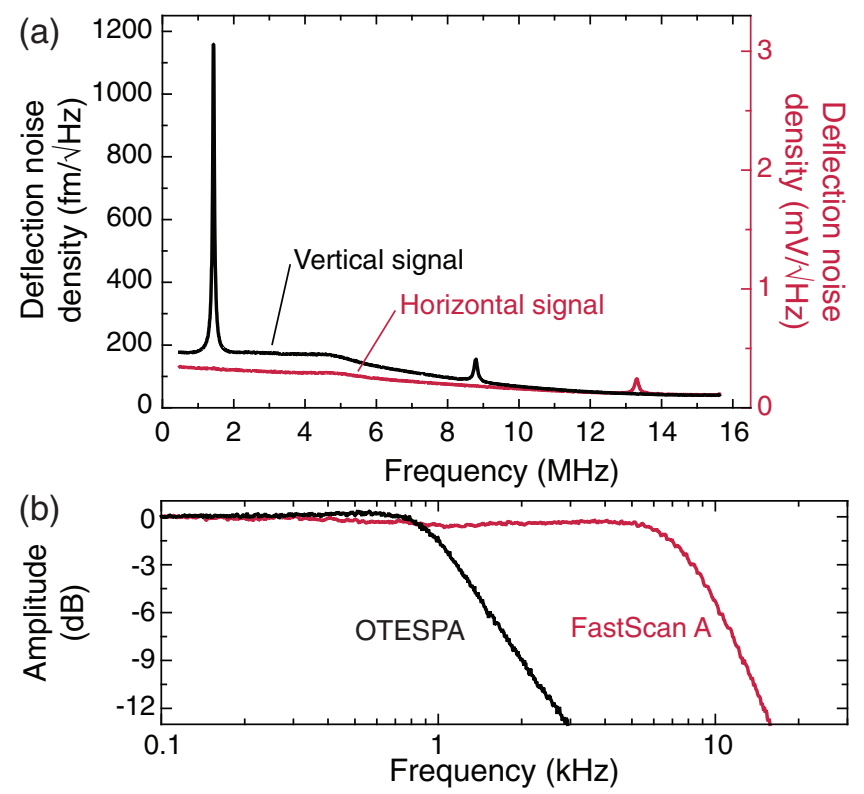

FIG. 4. (a) Thermal tune of a $2 \times 9 \mu \mathrm{m}$ small cantilever (Olympus BLAC10DS-A2). The vertical readout signal (left axis) shows the first and second resonance peaks at $1.4 \mathrm{MHz}$ and $8.8 \mathrm{MHz}$, respectively. The horizontal readout signal (right axis) shows the torsional resonance peak at 13.3 MHz. (b) Mechanical bandwidth measurement of large (OTESPA) and small (FastScan A) cantilevers in tapping mode.

respectively, as well as the torsional mode at $\simeq 13 \mathrm{MHz}$ on the horizontal readout signal for this cantilever. We see negligible levels of cross-talk between the two channels. Although our system is not optimized for low noise performance, we have measured baseline noise levels of our readout below $100 \mathrm{fm} / \sqrt{\mathrm{Hz}}$. While this level does not reach the excellent noise performance of OBD systems optimized for low noise performance, ${ }^{29-32}$ it is comparable to the noise levels we have measured for our commercial standard and small cantilever AFM systems (MultiMode and Dimension FastScan, Bruker Nano Surfaces).

Small cantilevers typically have a much higher resonance frequency compared to the large cantilevers that must be used in a standard AFM head, with a similar Q factor. This combination results in a much higher imaging bandwidth for small cantilevers. As an example, we measured the imaging bandwidth of a traditional large cantilever (OTESPA) and a small cantilever (FastScan A) using our AFM head. We measured the cantilever imaging bandwidth by sinusoidally modulating the sample height at variable frequency while measuring the cantilever oscillation amplitude, similar to methods described by Sulcheck et al. ${ }^{34}$ and Kokavecz et al. ${ }^{33}$ We glued a freshly-cleaved mica surface directly onto a piezo stack actuator (PL022.30, Physik Instrumente) driven by a highspeed piezo amplifier (Techproject, Vienna, Austria) for $z$ modulation. The cantilever amplitude was measured using a high-speed AFM controller (Anfatec, Oelsnitz, Germany). The modulation signal was generated with a lock-in amplifier (eLockIn204/2, Anfatec) and the cantilever amplitude recorded to the same unit. Sample height feedback was used with low gains only to prevent the cantilever from drifting away from the surface. The frequency at which the cantilever 
(a)

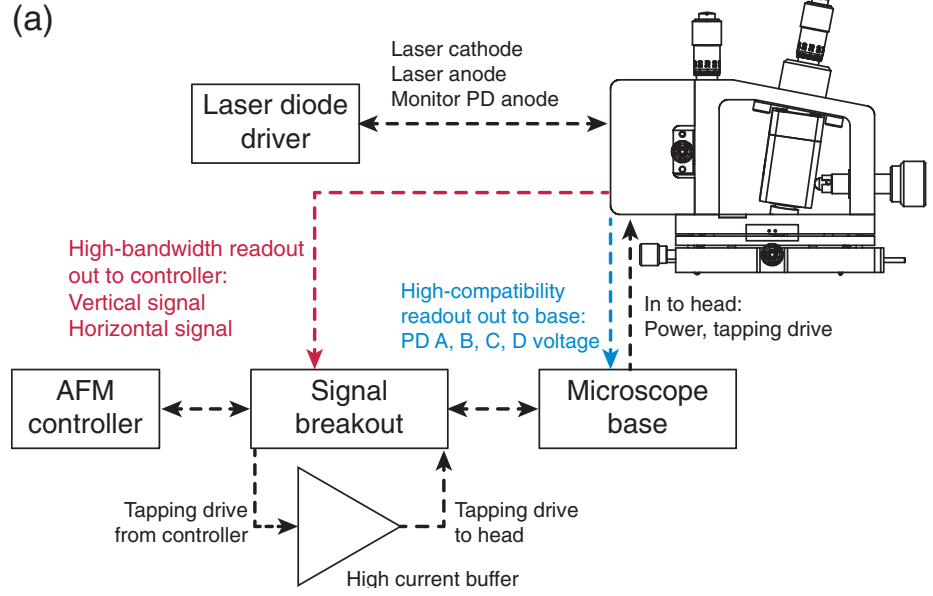

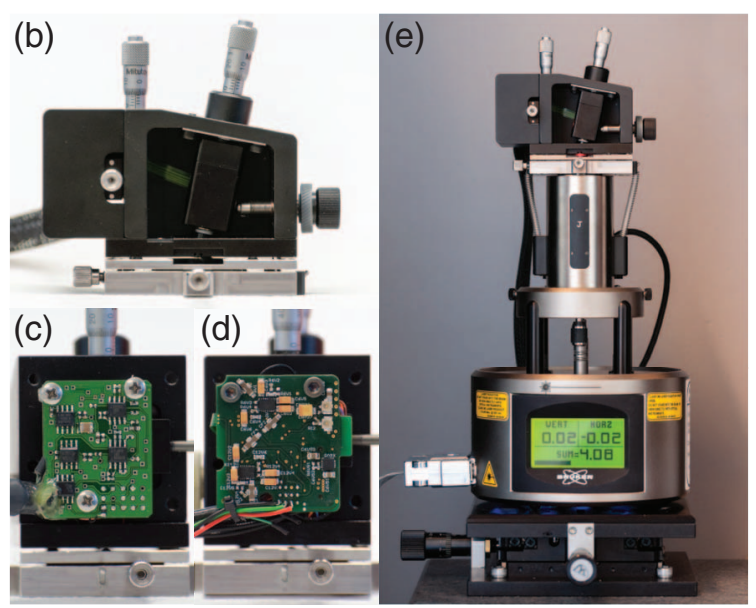

FIG. 5. (a) Schematic of the connections to interface the head with a standard AFM system. Photograph of the head (b), with electronics housing removed showing the high-compatibility readout (c) and the high-bandwidth readout (d). (e) Photograph of the head on top of a MultiMode AFM.

modulation amplitude fell to $-3 \mathrm{~dB}$ of the baseline value was defined as the bandwidth. The FastScan A cantilever has a measured bandwidth of $8 \mathrm{kHz}$, almost an order of magnitude higher than that of the OTESPA.

\section{INTEGRATION WITH THE AFM SYSTEM}

Our small cantilever head has been designed to easily integrate into a MultiMode AFM system. We use an external laser diode driver (LDX3412, ILX Lightwave, Irvine, CA) to provide current, monitor photodiode signal, and laser ground to the laser diode. In practice, we have found benefit in externally setting the laser power, for example, adjusting to a lower laser power on samples that are thermally sensitive. The head readout electronics connect to the MultiMode base through a 15 way Micro-D cable (Axon Cable, Montmirail, France). This cable provides power (analog ground, $\pm 15 \mathrm{~V}$ supply, laser ground and $\mathrm{a}+5 \mathrm{~V}$ supply referenced to the laser ground) and the tapping drive signal to the head. The laser grounds from the diode driver and from the microscope base are connected through a ferrite bead. The $+5 \mathrm{~V}$ supply is used to power a circuit (EL6204, Intersil, Milpitas, CA) for RF modulation of the laser diode drive at $500 \mathrm{MHz} .{ }^{30}$ For the high-compatibility readout, the voltage signals from the readout electronics are returned to the microscope base through the 15 way cable (Figure 5(a)). For the high-bandwidth readout, the horizontal and vertical deflection signals are calculated within the readout electronics and are sent directly to the AFM controller through a signal access module (Figure 5(b)). The signal access module is also used to send the internal tapping drive signal through the high-current buffer for driving the shaker stack piezo. Figure 5(c) shows a picture of our head mounted in place on top of a MultiMode V AFM. The translation stage is designed to mount directly on top of the standard scanners of the MultiMode system.

\section{HIGH-SPEED AFM IMAGING PERFORMANCE}

We evaluated the performance gain in the complete AFM system when using our AFM head by measuring the closed-loop bandwidth of the MultiMode AFM system with a Nanoscope V controller and EV scanner. We mounted a large cantilever (RTESP, Bruker AFM probes) in the standard MultiMode head, and a small cantilever (FastScan A) in our AFM head. An external lock-in amplifier (eLockIn205/2, Anfatec) generated a surface modulation signal that was added to the low voltage $z$ signal output from the signal access module. An external piezotube amplifier identical to the one in the Nanoscope V controller amplified the combined signal, which was then input into the signal access module as a highvoltage $z$ signal. We input the height signal generated by the Nanoscope controller via the front panel output into the external lock-in amplifier. We set the feedback gains using our AFM head by increasing the gains until just before the system became unstable during a $z$ modulation at $11 \mathrm{kHz}$, roughly the scanner resonance frequency. On the standard MultiMode head, we scaled the gains by the ratio of amplitude sensitivities to keep the feedback constant. Figure 6 shows the closedloop bandwidth amplitude (upper part) and phase (lower part) for both heads with their respective cantilevers. The amplitude plot shows clearly that the AFM system using the MultiMode head with the large cantilever is limited by the mechanical bandwidth of the cantilever. Beyond $2 \mathrm{kHz}$, the amplitude and phase response roll off sharply, due to both the rolloff of the controller and the roll-off of the cantilever (compare with the similar response from the large cantilever in Figure 4(b)). In contrast, with our AFM head and a small cantilever, the AFM system is limited by the scanner $z$ resonance. The roll-off visible from $1 \mathrm{kHz}$ to $7 \mathrm{kHz}$ is due to the controller, with gains set such that the system remains stable through the resonance peak of the scanner; the phase plot shows no additional roll-off until the scanner resonance. In this measurement, the FastScan A mechanical bandwidth limit of $\sim 10 \mathrm{kHz}$ is masked within the resonance of the scanner. We estimate a $5 \times$ increase in the controllable bandwidth using our AFM head with a FastScan A over the MultiMode head with RTESP based on a shift in the $-180^{\circ}$ point from $2.3 \mathrm{kHz}$ to $11 \mathrm{kHz}$. With the large cantilever, the speed limiting component is the mechanical bandwidth of the cantilever. With the small cantilever, the speed limiting component is 


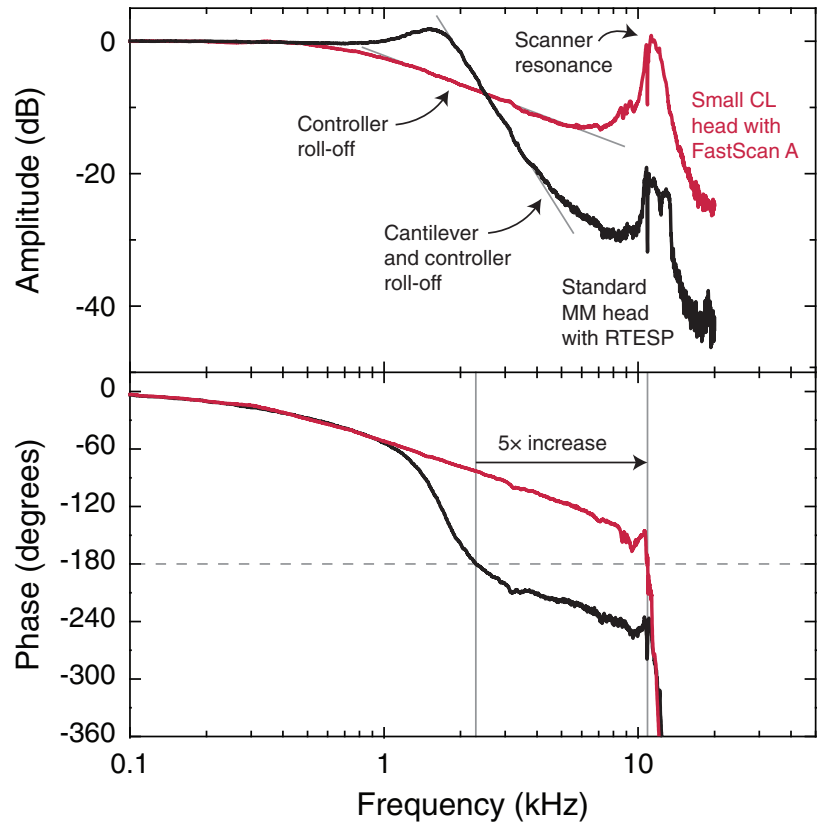

FIG. 6. Increase in the closed-loop bandwidth of the MultiMode AFM system using small cantilevers. The AFM system equipped with a standard head and a large cantilever (black curve) is cantilever limited, visible by the sharp roll-off in amplitude and phase beyond $2 \mathrm{kHz}$. The AFM system equipped with our AFM head and a small cantilever (red curve) is limited by the $z$ resonance of the scanner; the feedback is set to ensure stability through the resonance peak of the scanner. The increase in the $-180^{\circ}$ point from $2.3 \mathrm{kHz}$ to $11 \mathrm{kHz}$ suggests a 5-fold increase in the controllable bandwidth of the AFM system over the standard MultiMode.

the $z$ scanner resonance. Many approaches to overcome the $z$ scanner limitation are reported in literature. $6,8,14,16-19,23,24$ By employing such techniques together with our AFM head and small cantilevers, we expect a total bandwidth increase of $10 \times$ could be easily achieved, making again the FastScan A cantilever the speed limiting component.

To illustrate the speed improvements during imaging, we imaged a sample of Celgard (Celgard LLC, Charlotte, NC) in tapping mode in air. We used a MultiMode AFM with JV scanner and Nanoscope V controller. With a large cantilever for tapping mode in air (RTESPA) we find a significant degradation in the ability of the AFM system to track the topography above line scan rates of $1-2 \mathrm{~Hz}$ for a $1 \mu \mathrm{m}$ scan size. Using our small cantilever AFM head and a FastScan A cantilever, we can image the Celgard sample with good tracking at $10 \mathrm{~Hz}$ line-rate for a $1 \mu \mathrm{m}$ scan size (Figures 7(a) and 7(b) show the height and phase images, respectively). We used a small amount of rounding on the scan shape to limit excitation of scanner turnaround resonance. Because we have made no other system modification, the increase imaging speed can be directly attributed to the use of cantilevers with higher imaging bandwidth.

Our system provides a platform in which a few additional AFM system modifications can enable imaging at very high rates. In addition to the cantilever, the other two major components of the AFM system in terms of speed limits are the scanner resonances and the controller loop rate. To address the limitations of the scanner, we used an on-line system identification approach in order to compensate for the lateral scanner dynamics as described by Burns et al. ${ }^{21}$ We replaced the Nanoscope V controller with a high-speed AFM controller (AFT-MMC50, Anfatec) that was interfaced with our AFM head and a MultiMode EV scanner. We used small cantilevers with resonance frequency $\simeq 80 \mathrm{kHz}$ and $Q \simeq 3$ in fluid (SCL Sensor Tech, Vienna, Austria) which results in an expected cantilever imaging bandwidth $\sim 80 \mathrm{kHz}$. Finally, our sample was a mixed DLPC/DPPC lipid bilayer supported on a mica substrate. ${ }^{35,36}$ The small sample topography of a few nanometers limits excitation of the scanner $z$ resonance. Figure 7(b) shows height images of the lipid bilayer sample imaged in tapping mode at $100 \mathrm{~Hz}, 200 \mathrm{~Hz}$, and $500 \mathrm{~Hz}$ line-rates, with scan sizes of $4.3 \mu \mathrm{m}$ for the 100 and $200 \mathrm{~Hz}$ images and $2.7 \mu \mathrm{m}$ for the $500 \mathrm{~Hz}$ image. Even at these elevated scan rates, the AFM system is able to accurately track the surface without disrupting the lipid bilayer.

\section{CONCLUSIONS}

The factor that limits imaging speed in many standard AFM systems is the cantilever. Using small cantilevers on an otherwise unmodified AFM system permits imaging at higher speed. We have presented an AFM head for use with small cantilevers designed to be both easy to use, and easily integrated into a standard commercial MultiMode scanningsample AFM system. The modular nature of our design permits the exchange of the optical assembly and the readout electronics; we have demonstrated and evaluated two different designs for the optical assembly and signal readout electronics. The interested reader is encouraged to contact us regarding the construction of a copy of our AFM head for themselves. ${ }^{26}$ For integration into the AFM system, the only (a)

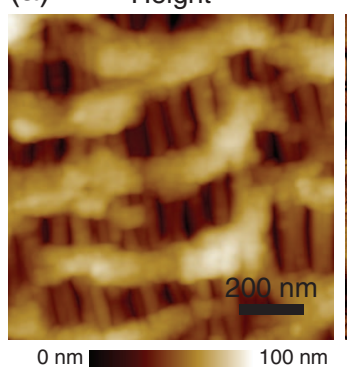

Phase

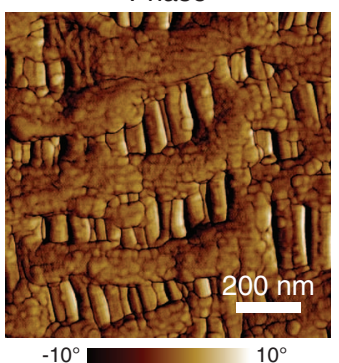

(b) 100 lines/s

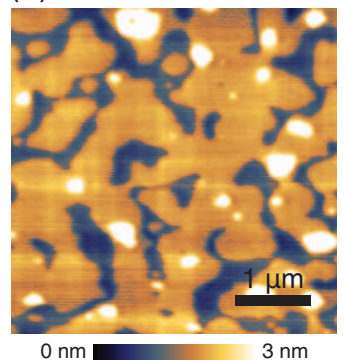

200 lines/s

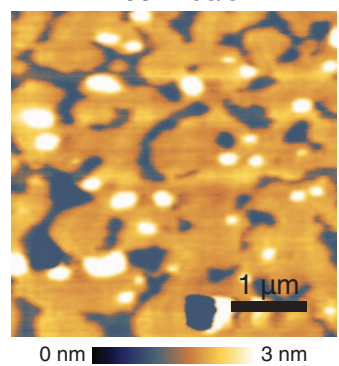

500 lines/s

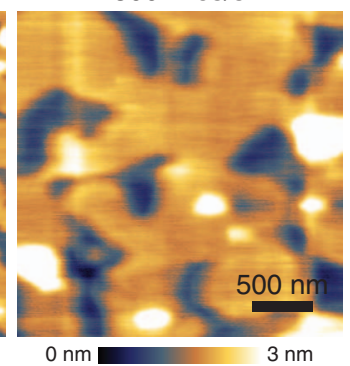

FIG. 7. (a) Imaging of Celgard in air using our AFM head with a FastScan A cantilever at $10 \mathrm{~Hz}$ line-rate on a MultiMode AFM with JV scanner and Nanoscope $\mathrm{V}$ controller. (b) High-speed tapping mode height images of DLPC/DPPC lipid bilayers at 100-500 Hz line-rate using our AFM head with small cantilevers in fluid, on-line compensation of the lateral scanner dynamics and a high-speed AFM controller. 
requirements for use are an external laser diode driver, and optionally, a signal access module. For the user, our design has approximately the same type and number of adjustments to be made for cantilever alignment as a standard AFM head and hence provides no greater difficulty in operation. Finally, we show that our AFM head, coupled with additional scanner resonance compensation and a high-speed controller, can image biological samples at very high scan rates.

\section{ACKNOWLEDGMENTS}

We thank the Atelier de l'institut de production et robotique at EPFL for fabrication of the mechanical components. This work has been funded by the European Union's Seventh Framework Programme FP7/2007-2011 under Grant Agreement No. 286146 and the European Union's Seventh Framework Programme FP7/2007-2013/ERC under Grant Agreement No. 307338, and the Swiss National Science Foundation through Grant Nos. 205321_134786 and 205320_152675 as well as co-funded by the State Secretariat for Education, Research and Innovation and the European Union under Grant No. E!8213 TRIPLE-S Microscope.

The authors declare no financial competing interests.

${ }^{1}$ S. Alexander, L. Hellemans, O. Marti, J. Schneir, V. Elings, P. K. Hansma, M. Longmire, and J. Gurley, "An atomic-resolution atomic-force microscope implemented using an optical lever," J. Appl. Phys. 65(1), 164-167 (1989).

${ }^{2}$ D. A. Walters, J. P. Cleveland, N. H. Thomson, P. K. Hansma, M. A. Wendman, G. Gurley, and V. Elings, "Short cantilevers for atomic force microscopy," Rev. Sci. Instrum. 67(10), 3583-3590 (1996).

${ }^{3}$ M. B. Viani, T. E. Schäffer, A. Chand, M. Rief, H. E. Gaub, and P. K. Hansma, "Small cantilevers for force spectroscopy of single molecules," J. Appl. Phys. 86(4), 2258-2262 (1999).

${ }^{4}$ T. Ando, T. Uchihashi, and T. Fukuma, "High-speed atomic force microscopy for nano-visualization of dynamic biomolecular processes," Prog. Surf. Sci. 83(7-9), 337-437 (2008).

${ }^{5}$ C. Richter, P. Weinzierl, W. Engl, C. Penzkofer, B. Irmer, and T. Sulzbach, "Cantilever probes for high speed AFM," Microsyst. Technol. 18(7-8), 1119-1126 (2012).

${ }^{6}$ T. Ando, N. Kodera, E. Takai, D. Maruyama, K. Saito, and A. Toda, "A high-speed atomic force microscope for studying biological macromolecules," Proc. Natl. Acad. Sci. U.S.A. 98(22), 12468-12472 (2001).

${ }^{7}$ P. K. Hansma, G. Schitter, G. E. Fantner, and C. Prater, "High-speed atomic force microscopy," Science 314(5799), 601-602 (2006).

${ }^{8}$ G. E. Fantner, G. Schitter, J. H. Kindt, T. Ivanov, K. Ivanova, R. Patel, N. Holten-Andersen, J. Adams, P. J. Thurner, I. W. Rangelow, and P. K. Hansma, "Components for high speed atomic force microscopy," Ultramicroscopy 106(8-9), 881-887 (2006).

${ }^{9}$ G. E. Fantner, R. J. Barbero, D. S. Gray, and A. M. Belcher, "Kinetics of antimicrobial peptide activity measured on individual bacterial cells using high-speed atomic force microscopy," Nat. Nanotechnol. 5(4), 280-285 (2010).

${ }^{10}$ N. Kodera, D. Yamamoto, R. Ishikawa, and T. Ando, "Video imaging of walking myosin $\mathrm{V}$ by high-speed atomic force microscopy," Nature 468(7320), 72-76 (2010).

${ }^{11}$ T. Uchihashi, R. Iino, T. Ando, and H. Noji, "High-speed atomic force microscopy reveals rotary catalysis of rotorless F1-ATPase," Science 333(6043), 755-758 (2011).

${ }^{12}$ T. Gutsmann, G. E. Fantner, J. H. Kindt, M. Venturoni, S. Danielsen, and P. K. Hansma, "Force spectroscopy of collagen fibers to investigate their mechanical properties and structural organization," Biophys. J. 86(5), 31863193 (2004)

${ }^{13}$ F. Rico, L. Gonzalez, I. Casuso, M. Puig-vidal, and S. Scheuring, "High-speed force spectroscopy molecular dynamics simulations," Science 342(6159), 741-743 (2013).

${ }^{14}$ I. S. Bozchalooi, K. Youcef-Toumi, D. J. Burns, and G. E. Fantner, "Compensator design for improved counterbalancing in high speed atomic force microscopy,” Rev. Sci. Instrum. 82(11), 113712 (2011).
${ }^{15}$ A. D. L. Humphris, M. J. Miles, and J. K. Hobbs, "A mechanical microscope: High-speed atomic force microscopy," Appl. Phys. Lett. 86(3), 034106 (2005).

${ }^{16}$ J. H. Kindt, G. E. Fantner, J. A. Cutroni, and P. K. Hansma, "Rigid design of fast scanning probe microscopes using finite element analysis," Ultramicroscopy 100(3-4), 259-265 (2004).

${ }^{17}$ H. Watanabe, T. Uchihashi, T. Kobashi, M. Shibata, J. Nishiyama, R. Yasuda, and T. Ando, "Wide-area scanner for high-speed atomic force microscopy," Rev. Sci. Instrum. 84(5), 053702 (2013).

${ }^{18}$ M. J. Rost, L. Crama, P. Schakel, E. van Tol, G. B. E. M. van VelzenWilliams, C. F. Overgauw, H. ter Horst, H. Dekker, B. Okhuijsen, M. Seynen, A. Vijftigschild, P. Han, A. J. Katan, K. Schoots, R. Schumm, W. van Loo, T. H. Oosterkamp, and J. W. M. Frenken, "Scanning probe microscopes go video rate and beyond," Rev. Sci. Instrum. 76(5), 053710 (2005).

${ }^{19}$ E. C. M. Disseldorp, F. C. Tabak, A. J. Katan, M. B. S. Hesselberth, T. H. Oosterkamp, J. W. M. Frenken, and W. M. van Spengen, "MEMS-based high speed scanning probe microscopy," Rev. Sci. Instrum. 81(4), 043702 (2010).

${ }^{20}$ Y. Wu, J. Shi, C. Su, and Q. Zou, "A control approach to cross-coupling compensation of piezotube scanners in tapping-mode atomic force microscope imaging,” Rev. Sci. Instrum. 80(4), 043709 (2009).

${ }^{21}$ D. J. Burns, K. Youcef-Toumi, and G. E. Fantner, "Indirect identification and compensation of lateral scanner resonances in atomic force microscopes," Nanotechnology 22(31), 315701 (2011).

${ }^{22}$ N. Kodera, M. Sakashita, and T. Ando, "Dynamic proportional-integraldifferential controller for high-speed atomic force microscopy," Rev. Sci. Instrum. 77(8), 083704 (2006).

${ }^{23}$ G. Schitter, P. Menold, H. F. Knapp, F. Allgower, and A. Stemmer, "High performance feedback for fast scanning atomic force microscopes," Rev. Sci. Instrum. 72(8), 3320 (2001).

${ }^{24}$ G. Schitter, F. Allgöwer, and A. Stemmer, "A new control strategy for highspeed atomic force microscopy," Nanotechnology 15(1), 108-114 (2004).

${ }^{25} \mathrm{~J}$. Mertz, O. Marti, and J. Mlynek, "Regulation of a microcantilever response by force feedback," Appl. Phys. Lett. 62(19), 2344-2346 (1993).

${ }^{26}$ We invite those interested to contact us (georg.fantner@epfl.ch) for assistance in building a copy of the AFM head for non-commercial purposes.

${ }^{27}$ M. B. Viani, T. E. Schaffer, G. T. Paloczi, L. I. Pietrasanta, B. L. Smith, J. B. Thompson, M. Richter, M. Rief, H. E. Gaub, K. W. Plaxco, A. N. Cleland, H. G. Hansma, and P. K. Hansma, "Fast imaging and fast force spectroscopy of single biopolymers with a new atomic force microscope designed for small cantilevers,” Rev. Sci. Instrum. 70(11), 4300 (1999).

${ }^{28}$ P. K. Hansma, B. Drake, J. Thompson, J. H. Kindt, and D. Hale, "Measurement head for atomic force microscopy and other applications," U.S. patent 6,871,527 B2 (29 March 2005).

${ }^{29}$ R. Enning, D. Ziegler, A. Nievergelt, R. Friedlos, K. Venkataramani, and A. Stemmer, "A high frequency sensor for optical beam deflection atomic force microscopy," Rev. Sci. Instrum. 82(4), 043705 (2011).

${ }^{30}$ T. Fukuma, M. Kimura, K. Kobayashi, K. Matsushige, and H. Yamada, "Development of low noise cantilever deflection sensor for multienvironment frequency-modulation atomic force microscopy," Rev. Sci. Instrum. 76(5), 053704 (2005).

${ }^{31}$ T. Fukuma and S. P. Jarvis, "Development of liquid-environment frequency modulation atomic force microscope with low noise deflection sensor for cantilevers of various dimensions," Rev. Sci. Instrum. 77(4), 043701 (2006).

${ }^{32}$ T. Fukuma, "Wideband low-noise optical beam deflection sensor with photothermal excitation for liquid-environment atomic force microscopy," Rev. Sci. Instrum. 80(2), 023707 (2009).

${ }^{33}$ J. Kokavecz, O. Marti, P. Heszler, and Á. Mechler, "Imaging bandwidth of the tapping mode atomic force microscope probe," Phys. Rev. B 73(15), 155403 (2006).

${ }^{34}$ T. Sulchek, G. G. Yaralioglu, C. F. Quate, and S. C. Minne, "Characterization and optimization of scan speed for tapping-mode atomic force microscopy,” Rev. Sci. Instrum. 73(8), 2928-2936 (2002).

${ }^{35}$ A. Mecke, S. Uppuluri, T. M. Sassanella, D.-K. Lee, A. Ramamoorthy, J. R. Baker, B. G. Orr, and M. M. Banaszak Holl, "Direct observation of lipid bilayer disruption by poly(amidoamine) dendrimers," Chem. Phys. Lipids 132(1), 3-14 (2004).

${ }^{36}$ B. Erickson, S. C. DiMaggio, D. G. Mullen, C. V. Kelly, P. R. Leroueil, S. A. Berry, J. R. Baker, B. G. Orr, and M. M. Banaszak Holl, "Interactions of poly(amidoamine) dendrimers with Survanta lung surfactant: the importance of lipid domains," Langmuir 24(19), 11003-11008 (2008). 